imaging (MRI) - might distinguish surgical responders from early non-responders. Methods: We retrospectively identified $35 \mathrm{TN}$ patients treated surgically from 2005-2017 with high-resolution, pre-operative MRI scans adequate for quantitative structural analysis. Patients were classified as non-responders if, within 12-months after surgery, they: 1) underwent or were offered another surgical procedure; or 2) reported persistent, inadequately-controlled pain. Volumes of pain-relevant subcortical structures (amygdala, thalamus, and hippocampus) were measured on T1-weighted MRI scans using an automated approach (FSL-FIRST). Results: Surgical responders had significantly larger hippocampi bilaterally compared to early non-responders. Thalamus and amygdala volumes did not differ between groups. Conclusions: Pre-operative differences in brain structure, notably in the hippocampus, may predict durability of response to surgery in patients with TN.

Table 1: Demographic and Clinical Characteristics of TN Patients:

\begin{tabular}{|l|c|c|c|}
\hline & Responders & $\begin{array}{c}\text { Non-Re- } \\
\text { sponders }\end{array}$ & $\begin{array}{c}\text { P-value } \\
\text { (2-tailed) }\end{array}$ \\
\hline Outcome Group & 23 & 12 & N/A \\
\hline Sex (Female/Male) & $9 / 14$ & $6 / 6$ & 0.5591 \\
\hline Age, years & $4.35 \pm 11.36$ & $53.75 \pm 16.33$ & 0.9111 \\
\hline Affected Side (Left/Right) & $5 / 18$ & $5 / 7$ & 0.2630 \\
\hline \# of Previous treatments & $0.13 \pm 0.34$ & $1.42 \pm 1.40$ & $0.0105^{*}$ \\
\hline $\begin{array}{l}\text { Surgery Performed (MVD/ } \\
\text { PRR) }\end{array}$ & $21 / 2$ & $10 / 2$ & 0.9722 \\
\hline Volumetric Assessment: & \multicolumn{3}{|l|}{} \\
\hline \multicolumn{4}{|l|}{ Respond- } \\
ers (mm3) & Responders & $\begin{array}{c}\text { P-value } \\
\text { (mm3) }\end{array}$ \\
\hline Hippocailed) \\
\hline Ipsilateral & & & \\
\hline Contralateral & $3440 \pm 365$ & $697 \pm 318$ & $0.0415^{*}$ \\
\hline Left & $3381 \pm 375$ & $3727 \pm 215$ & $0.0015^{*}$ \\
\hline Right & $3357 \pm 373$ & $3669 \pm 231$ & $0.0046^{*}$ \\
\hline * p<0.05 & $3464 \pm 361$ & $3754 \pm 301$ & $0.0178^{*}$ \\
\hline $\begin{array}{l}\text { MVD - microvascular decompression surgery } \\
\text { PRR - percutaneous retrogasserian rhizotomy }\end{array}$ \\
\hline Values are mean +/- standard deviation where appropriate \\
\hline
\end{tabular}

\section{Neuromuscular Disease and EMG}

\section{P.069}

\section{Respiratory dysfunction and sleep disordered breathing in children with Myasthenia Gravis}

HF Qashqari (Toronto)* I Narang (Toronto) H Katzberg (Toronto) K Vezina (Toronto) A Khayat (Toronto) N Chrestian (Toronto) J Vajsar (Toronto)

doi: 10.1017/cjn.2018.171

Background: Myasthenia Gravis ( MG) is an autoimmune disease that affects the neuromuscular junction. It typically presents with fluctuating muscle weakness which can affect respiratory muscles. Data about the prevalence of sleep disordered breathing in children with MG and the benefits of non-invasive ventilation outside the setting of MG crisis has not been studied so far. Methods: Eleven children between 3 and 18 years old with confirmed MG were recruited from the The Hospital for Sick Children Neuromuscular clinic in a prospective observational study. Informed consent was obtained and patients underwent PFTs, MIP/MEP, SNIP, FVC and standard polysomnography testing's. Results: In our study, we found that 2/11 children had abnormal Apnea Hypopnea index (AHI) and were diagnosed with obstructive sleep apnea (OSA). One of them has juvenile ocular MG with mild to moderate OSA and the second child has congenital MG with mild OSA. CPAP therapy was initiated for both patients. Conclusions: In our cohort, obstructive sleep apnea rate was significantly higher in children with MG than the known prevalence in general pediatric population ( $18 \%$ vs $2-3 \%$ ). Early diagnosis and management of OSA can have great impact on children's health and quality of life. A larger study is needed to validate our findings.

\section{P.070}

\section{Autosomal dominant MARS mutation linked to severe early onset CMT2U}

HJ McMillan (Ottawa)* MK Gillespie (Ottawa) KD Kernohan (Ottawa) $R$ Myer-Schuman (Ann Arbor) A Antonellis (Ann Arbor) KM Boycott (Ottawa)

doi: 10.1017/cjn.2018.172

Background: Methionyl-tRNA synthetase (MARS) links methionine to its cognate tRNA required for translation. MARS mutations have been linked to adult-onset CMT2U. Methods: The proband had weakness in her first year of life, sitting at 11 months and walking at 20 months old. At 4 years old she was areflexic with distal > proximal weakness. Nerve conduction studies showed normal median and sural sensory responses with absent common peroneal, low median and tibial motor amplitudes. EMG noted denervation and quadriceps biopsy revealed neurogenic atrophy. Genetic testing for spinal muscular atrophy and sequencing of MNF2, RAB7A, LMNA, MPZ, HSPB1, NEFL, GADP1, TRPV4, HSPB8, GJB1 and PLEK8G5 were negative. She stopped walking at 9 years old and could not raise her arms above her head at 11 years old. Results: Exome sequencing identified MARS: c.1189G>A; p.Ala397Thr. To determine the functional consequences of p.A397T-MARS, yeast complementation assays were performed. Wild type or mutant MARS were cloned into yeast lacking the endogenous MARS ortholog. Wild-type MARS 